\title{
Identifying optimal parameters for infrared neural stimulation in the peripheral nervous system
}

\author{
Graham Throckmorton,, a,b Jonathan Cayce, ${ }^{\text {a,b }}$ Zane Ricks, \\ Wilson R. Adams ${ }^{\circ},{ }^{\text {a,b }}$ Eric Duco Jansen, ${ }^{\text {a,b,c }}$ and Anita Mahadevan-Jansen ${ }^{\text {a,b,c, } * *}$ \\ ${ }^{a}$ Vanderbilt Biophotonics Center, Keck FEL Center, Nashville, Tennessee, United States \\ ${ }^{b}$ Vanderbilt University, Department of Biomedical Engineering, Nashville, Tennessee, \\ United States \\ ${ }^{c}$ Vanderbilt University Medical Center, Department of Neurological Surgery, Nashville, \\ Tennessee, United States
}

\begin{abstract}
Significance: Infrared neural stimulation (INS) utilizes pulsed infrared light to selectively elicit neural activity without exogenous compounds. Despite its versatility in a broad range of biomedical applications, no comprehensive comparison of factors pertaining to the efficacy and safety of INS such as wavelength, radiant exposure, and optical spot size exists in the literature.

Aim: Here, we evaluate these parameters using three of the wavelengths commonly used for INS, $1450 \mathrm{~nm}, 1875 \mathrm{~nm}$, and $2120 \mathrm{~nm}$.

Approach: In an in vivo rat sciatic nerve preparation, the stimulation threshold and transition rate to $100 \%$ activation probability were used to compare the effects of each parameter.

Results: The pulsed diode lasers at $1450 \mathrm{~nm}$ and $1875 \mathrm{~nm}$ had a consistently higher $\left(\sim 1.0 \mathrm{~J} / \mathrm{cm}^{2}\right)$ stimulation threshold than that of the Ho:YAG laser at $2120 \mathrm{~nm}\left(\sim 0.7 \mathrm{~J} / \mathrm{cm}^{2}\right)$. In addition, the Ho:YAG produced a faster transition rate to $100 \%$ activation probability compared to the diode lasers. Our data suggest that the superior performance of the Ho:YAG is a result of the high-intensity microsecond spike at the onset of the pulse. Acute histological evaluation of diode irradiated nerves revealed a safe range of radiant exposures for stimulation.
\end{abstract}

Conclusion: Together, our results identify measures to improve the safety, efficacy, and accessibility of INS technology for research and clinical applications.

(C) The Authors. Published by SPIE under a Creative Commons Attribution 4.0 Unported License. Distribution or reproduction of this work in whole or in part requires full attribution of the original publication, including its DOI. [DOI: 10.1117/1.NPh.8.1.015012]

Keywords: infrared neural stimulation; efficacy; clinical translation; peripheral nerves; in vivo; neurophotonics.

Paper 20071R received Sep. 28, 2020; accepted for publication Feb. 17, 2021; published online Mar. 31, 2021.

\section{Introduction}

For the last century, scientists and clinicians have used the direct application of electrical current to modulate neural activity and probe the structure and function of the nervous system. ${ }^{1-4}$ To this day, electrical stimulation remains the gold standard for assessing and quantifying neural activity. Despite its broad utility, electrical stimulation is hindered by fundamental limitations, namely, current spread and stimulation artifacts. Current spread, in which the injected current disperses into the surrounding tissue, restricts the spatial specificity of neural activation. ${ }^{5,6}$ Spatial specificity is both clinically and experimentally advantageous when targeting specific regions of a nerve or other neural structures such as the brain and cochlea which have a high degree of spatial dependence. ${ }^{7-9}$ Clinically, current spread in deep brain stimulation cases can cause deleterious side effects that may hinder the effective treatment of Parkinson's disease. ${ }^{10-12}$

*Address all correspondence to Anita Mahadevan-Jansen, anita.mahadevan-jansen@vanderbilt.edu 
In the peripheral nervous system, current spread can directly induce muscle contractions that may falsely indicate nerve viability in damaged nerve segments. ${ }^{13-15}$ Improving the spatial specificity and overcoming current spread requires precise control over electrode placement and/or necessitates direct electrical coupling to the neuron, as done in patch-clamp techniques. In addition to current spread, electrical stimulation artifacts impede the ability to record proximal to the stimulation site because the artifact can mask the true electrophysiological signal. ${ }^{6}$ Stimulation artifacts can also impair the interpretation of nerve graft viability and of electrophysiological recordings during intraoperative nerve monitoring. ${ }^{5,16,17}$ Thus, neural stimulation techniques providing spatially confined excitation without stimulation artifacts represent highly desired tools in both clinical and experimental settings.

Initial studies published on infrared neural stimulation (INS), ${ }^{5,18-20}$ along with the foundational work in optogenetics, ${ }^{21}$ demonstrated the ability to optically stimulate selective neuronal populations without stimulation artifacts or tissue contact. While optogenetics relies on the genetic modification of the neural tissue to express light-sensitive ion channels, INS intrinsically elicits neural through tissue absorption of short-wave infrared (SWIR) light. ${ }^{18}$ Moreover, the inherent spatial precision of INS is a direct result of the rapid spatiotemporal thermal gradient INS creates. ${ }^{18}$ The thermal energy from the infrared pulses is spatially confined to the irradiated volume as determined by the laser spot size and the penetration depth of the light in tissue. The work of Shapiro et al. further shows how the deposition of thermal energy generates an action potential. ${ }^{22}$

During INS, the transient heating induced by water absorption causes a rapid change in cell membrane capacitance leading to the initiation of an action potential. ${ }^{22}$ In a subsequent study, Plaksin et al. showed that a thermal-mechanical effect causes the membrane to thin axially and expand laterally producing the change in cell membrane capacitance. ${ }^{23}$ Though researchers still debate the exact mechanism, this capacitive change appears to evoke action potentials through a universal mechanism triggered by thermal-mechanical effects within the extracellular phospholipid bilayer. ${ }^{22-28}$ Despite the incomplete understanding of the biophysical mechanism of INS, the innate label-free nature and high degree of spatial specificity of this technique make INS well suited for clinical translation.

Previously, the feasibility of INS in humans was demonstrated by activating specific functional connections in human dorsal rootlets more precisely than electrical stimulation. ${ }^{29}$ In the central nervous system, researchers have used INS for cortical mapping of the non-human primate visual system and modulating task-specific behavior. ${ }^{8,9,30-32}$ The application of INS in animal models for surgical guidance includes use in the prostate, bladder, and base of the skull to minimize collateral nerve damage that can give rise to urinary incontinence, sexual impotence, and facial paralysis, respectively. ${ }^{16,17,33-35}$ Together, these studies substantiate INS as a potentially valuable clinical tool in both the central and peripheral nervous systems.

Despite its proven capability as a clinical tool, the cost and size of lasers systems used for INS present a major barrier to its widespread use. Initially, INS studies used bulky and expensive flashlamp-pumped solid state lasers. As the field progressed, cheaper diode laser systems became more widely adopted in laboratories utilizing INS. Using these lower-cost diode systems $(\sim \$ 20 \mathrm{k})$, multiple groups published results demonstrating the efficacy of $1875 \mathrm{~nm}$ and $1450 \mathrm{~nm}$ light for eliciting neural activity. ${ }^{9,22,26,27,30-32,36-51}$ Custom diode systems can be made for a few thousand dollars but require some proficiency in laser hardware and electronics. Other groups opted to use fiber lasers with tunable thulium fiber lasers centered around $2000 \mathrm{~nm}$ being the most common, but this wavelength may be too strongly absorbed by water in the tissue. ${ }^{52-56}$ Many groups chose to construct custom laser systems to study INS, which largely accounts for the variability in parameters used throughout the literature. To make INS an accessible and reliable tool for clinical neural modulation, a need exists to evaluate the optimal laser parameters that result in safe and effective stimulation, including pulse duration, spot size, and radiant exposure. In addition, despite the multitude of studies performed using $1450 \mathrm{~nm}$ light, no histological evaluation of the safety for INS at $1450 \mathrm{~nm}$ exists. However, no histological or functional damage was observed in the acute studies using the Ho:YAG laser at $2120 \mathrm{~nm}$ or diode lasers at $1875 \mathrm{~nm} \cdot{ }^{29,31,57,58}$ A safety ratio (the ratio of the ablation threshold to stimulation threshold) of 2:1 was established..$^{29,57}$ Due to the thermal gradient inherent to INS, histological safety represents a crucial consideration for future experimental and translational efforts. ${ }^{29,31,56,57}$ 
The goal of this study is to identify optimal stimulation parameters across three SWIR wavelength lasers, two diode lasers centered at $1450 \mathrm{~nm}$ and $1875 \mathrm{~nm}$, and the "gold standard" Ho: YAG laser at $2120 \mathrm{~nm}$, to empirically evaluate the efficacy and histological safety of these wavelengths in an in vivo rat sciatic nerve model. The effect of spot size, pulse width, and radiant exposure on the efficacy and safety of INS was investigated. Our results identify strategies to maximize the efficacy and safety of diode lasers for INS while improving the accessibility and utility of INS for future research and clinical applications.

\section{Materials and Methods}

All experiments were conducted at the Vanderbilt Biophotonics Center in adherence to protocols approved by the Vanderbilt Institution of Animal Care and Use Committee.

\subsection{Animal Preparation}

In vivo sciatic nerve experiments were performed using adult male Sprague-Dawley rats ( $n=26,300$ to $350 \mathrm{~g}$ ) as a mammalian model. Animals were anesthetized by inhalation of isoflurane $(3 \%, 3 \mathrm{~L} / \mathrm{min})$ and maintained under sedation $(2 \%$ to $2.5 \%, 1.5 \mathrm{~L} / \mathrm{min})$ for the duration of the experiments. Once anesthetized, animals were placed on a polycarbonate platform and fitted with a nose cone to maintain anesthesia. A water-circulating heating pad (catalog\# 40-90-8, FHC, Bowdoin, Maine) was used to maintain a body temperature of $36^{\circ} \mathrm{C}$ to $37^{\circ} \mathrm{C}$ throughout experiments while the animal's body temperature was monitored using a rectal probe. Both hind limbs were shaved, and the dorsal surface of the feet were secured to the edge of the platform. A 3-cm incision was made posterior-laterally extending from the gluteus muscles to the popliteal region. The skin was separated from the underlying tissue to expose the biceps femoris, which was carefully incised to expose the sciatic nerve (Fig. 1). The muscle fascia overlying the surface of the nerve was delicately removed to expose the nerve surface. The epineurium was left intact. Nerves were periodically bathed in room temperature sterile saline throughout experiments to maintain tissue hydration and prevent desiccation. After the experiment, each rat was euthanized via anesthetic overdose followed by cervical dislocation that adhered to the IACUC approved protocol.

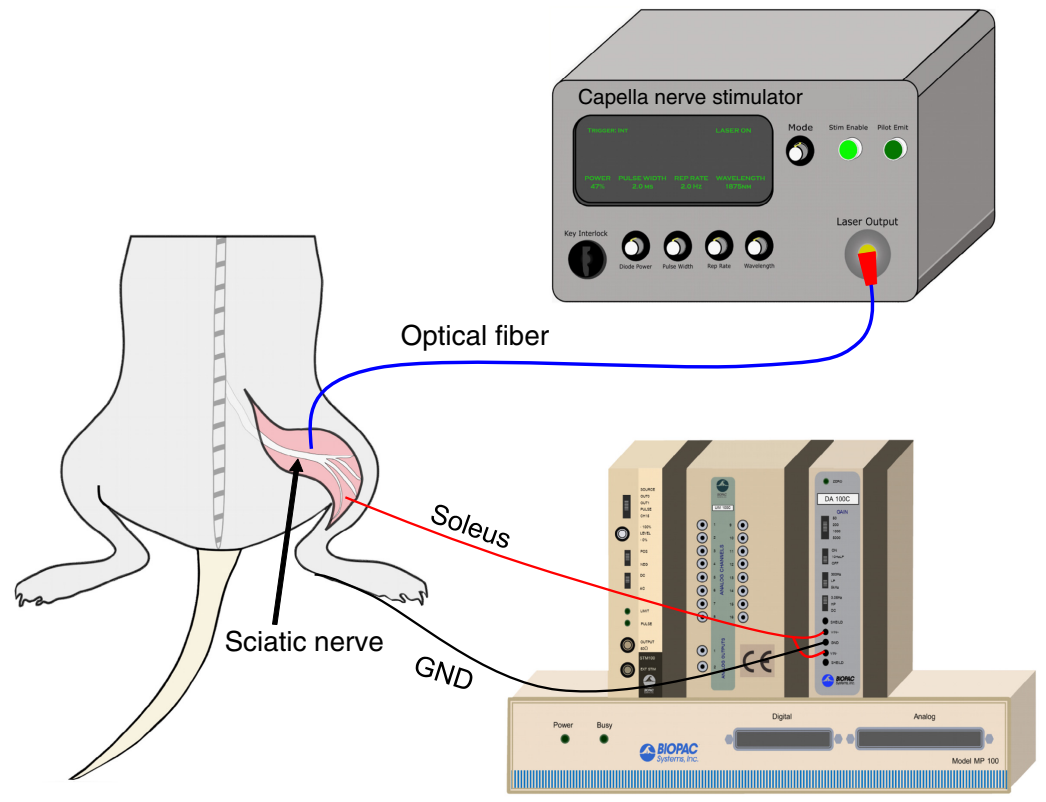

Fig. 1 Experimental setup for in vivo INS of the rat sciatic nerve. The laser source was coupled directly into a multimode optical fiber positioned over the nerve. The nerve was stimulated optically and CMAPs were recorded from the soleus muscle using a modular data acquisition system. 


\subsection{Electrophysiology}

Compound muscle action potentials (CMAPs) were recorded from paired, bipolar subdermal needle electrodes (Medtronic Xomed, Jacksonville, Florida) inserted into the soleus. A subdermal grounding electrode was also inserted into the foot of the same leg. CMAPs and the trigger output of the laser were recorded simultaneously with a modular data acquisition system (MP100, Biopac Systems Inc., Santa Barbara, California) using Acknowledge ${ }^{\circledR}$ software (Biopac Systems Inc., Santa Barbara, California). CMAPs were sampled at $6500 \mathrm{~Hz}$ and captured digitally after amplification using a differential amplifier (DA 100C, Biopac Systems Inc., Santa Barbara, California). All signals were amplified 1000x and bandpass filtered from 0.05 to $5000 \mathrm{~Hz}$. Before each experiment, the viability of the sciatic nerve was assessed with electrical stimulation ( 0.3 to $0.4 \mathrm{~V}$ at $500 \mu \mathrm{s}$ ) using a Prass standard monopolar stimulator probe (Medtronic Xomed, Jacksonville, Florida). Only CMAPs occurring within $5 \mathrm{~ms}$ of the infrared pulse were included in data analysis. Representative CMAP traces can be found in Fig. S1 in the Supplemental Materials.

\subsection{Infrared Neural Stimulation}

A parametric assessment of stimulation thresholds was performed with a total of four SWIR wavelengths and three spot sizes across a range of pulse widths and radiant exposures (Table 1). The lasers used for these experiments included two laser diode systems at $1450 \mathrm{~nm}$ and $1875 \mathrm{~nm}$, respectively (Capella, Lockheed Martin-Aculight, Bothell, Washington), a custom laser diode system at $1470 \mathrm{~nm}$ (Innovative Photonics Solutions, Monmouth Junction, New Jersey), and a Ho: YAG laser at $2120 \mathrm{~nm}$ (Model 1-2-3 laser, Schwartz Electro Optics, Inc., Concord, Massachusetts).

\subsubsection{Radiant exposure and pulse duration}

For all diode laser experiments at a given spot size, the diode current was adjusted to vary the radiant exposure between 0 and $2.5 \mathrm{~J} / \mathrm{cm}^{2}$. In experiments with the $1875 \mathrm{~nm}$ diode laser, the pulse duration was modulated for a given spot size to produce the necessary radiant exposures (e.g., $2 \mathrm{~ms}$ pulses for $500 \mu \mathrm{m}$ spot size and $5 \mathrm{~ms}$ pulses for $1000 \mu \mathrm{m}$ ). For the Ho:YAG laser, the radiant exposures were modulated with attenuators placed in the beam path. For each experimental trial, nerves were irradiated with a 10 -s pulse train at $2 \mathrm{~Hz}$ for a total of 20 pulses at a randomized radiant exposure.

Due to the limited power output of the $1450 \mathrm{~nm}$ and $1875 \mathrm{~nm}$ diode lasers, the $1470 \mathrm{~nm}$ diode laser was used to generate $350 \mu$ s pulses with equal energy to that of the Ho:YAG laser. The $1470 \mathrm{~nm}$ diode laser was only used to examine the effects of the pulse width. All pulse widths refer to the full width at half maximum of the pulse.

\subsubsection{Spot size}

Stimulation thresholds were determined for three spot sizes: $500 \mu \mathrm{m}(504.5 \pm 20 \mu \mathrm{m}), 800 \mu \mathrm{m}$ $(805.6 \pm 18.8 \mu \mathrm{m})$, and $1000 \mu \mathrm{m}(1019 \pm 29.8 \mu \mathrm{m})$ (Fig. 2). All spot sizes were measured using

Table 1 INS parameters.

\begin{tabular}{lccccc}
\hline \hline Laser & $\begin{array}{c}\text { Wavelength } \\
(\mathrm{nm})\end{array}$ & $\begin{array}{c}\text { Water absorption } \\
\text { coefficient }{ }^{\mathrm{a}}\left(\mathrm{cm}^{-1}\right)\end{array}$ & $\begin{array}{c}\text { Pulse } \\
\text { width }(\mu \mathrm{s})\end{array}$ & $\begin{array}{c}\text { Spot size } \\
(\mu \mathrm{m})\end{array}$ & $\begin{array}{c}\text { Radiant exposures } \\
\left(\mathrm{J} / \mathrm{cm}^{2}\right)\end{array}$ \\
\hline Diode & 1450 & 29.7 & 2000 & 500 to 1000 & 0 to 2.5 \\
& 1470 & 24.9 & 350 & 500 & 0 to 2.5 \\
& 1875 & 25.4 & $2000,3000,5000$ & 500 to 1000 & 0 to 2.5 \\
Ho:YAG & 2120 & 24 & $350^{\mathrm{b}}$ & 500 to 1000 & 0 to 1.0 \\
\hline \hline
\end{tabular}

${ }^{a}$ Water absorption coefficients are based on the cubic interpolation of data acquired by Hale and Querry $(1973) .^{59}$

${ }^{\mathrm{b}}$ Full width half maximum (FWHM). 


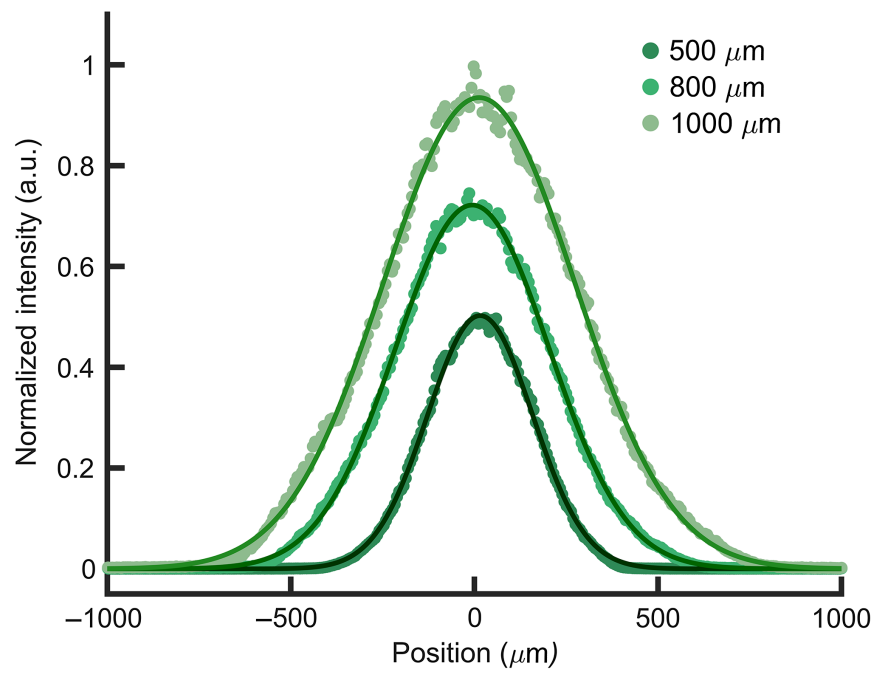

Fig. 2 Spot size measurements made using IR beam profiler fitted to a Gaussian distribution (solid lines). Maximum intensities are modulated for clear visualization. Spot Size $500=528.5 \mu \mathrm{m}, R^{2}=$ 0.9989; Spot Size $800=802.1 \mu \mathrm{m}, R^{2}=0.9993 ;$ Spot Size $1000=1003 \mu \mathrm{m}, R^{2}=0.9986$.

an infrared beam profiler (BP209-IR2, Thorlabs, Newton, New Jersey), validated using the knife-edge technique, ${ }^{60}$ and refer to the $1 / e^{2}$ diameter of the beam profile. Spot sizes were modulated by adjusting the distance between the bare $400 \mu \mathrm{m}$ diameter optical fiber (NA $=0.22$; Ocean Optics, Dunedin, Florida) output and the nerve surface. For the 500, 800, and $1000 \mu \mathrm{m}$ spot sizes, the average distance away from the nerve surface was $862.9 \pm 21.79,1874 \pm 156$, and $2646 \pm 240 \mu \mathrm{m}$, respectively. The fiber probes were positioned using a micromanipulator (World Precision Instruments, Sarasota, Florida) orthogonal to the main trunk of the sciatic nerve.

\subsubsection{Spikeless Ho:YAG pulse}

To investigate the effects of the leading spike in the Ho:YAG pulse on INS efficacy [Fig. 3(a)], the spike was eliminated using a $200-\mu \mathrm{m}$-thick glass chamber filled with deionized water placed just outside the laser chamber in the beam path. To achieve spikeless pulses, the pulse energy of the Ho:YAG was increased until the initial microsecond spike at the beginning of the pulse contained the necessary energy to create a vapor bubble. ${ }^{61}$ Once the bubble was generated from the

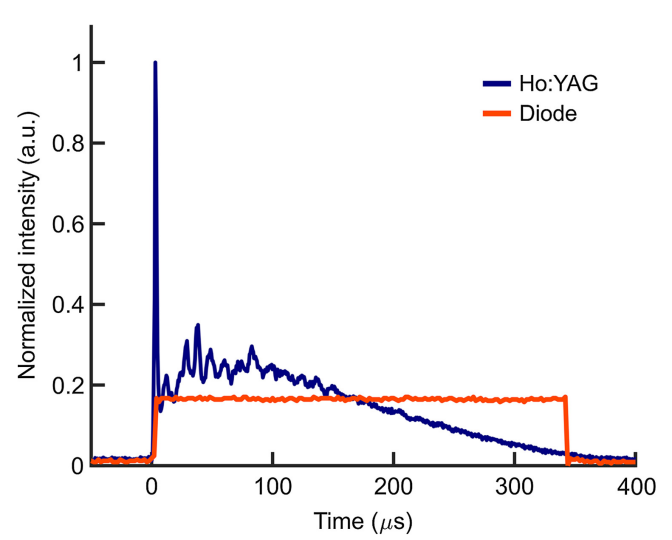

(a)

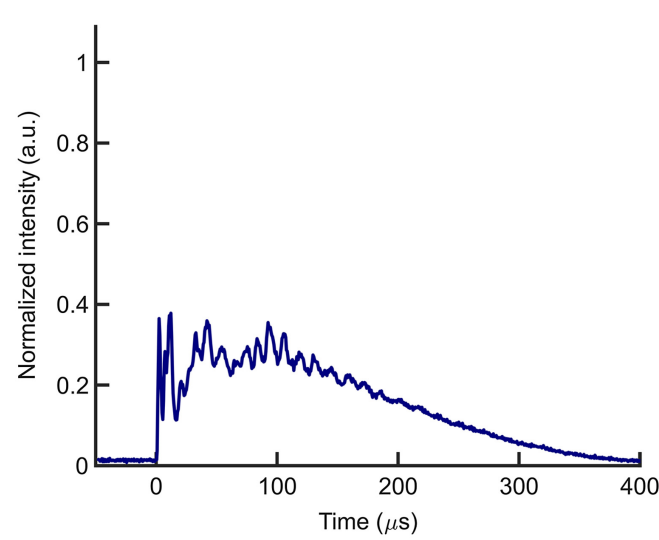

(b)

Fig. 3 AUC-normalized temporal pulse shapes from Ho:YAG and diode lasers. (a) AUC-normalized temporal pulse shapes from a regular Ho:YAG and $350 \mu$ s diode pulse. (b) AUC-normalized temporal pulse shape from a spikeless Ho:YAG pulse. Pulse traces were taken with amplified, InGaAs detector (PDA10D, Thorlabs, Newton, New Jersey). 
spike's energy, the spikeless remainder of the Ho:YAG pulses was able to pass through the vapor with minimal absorption and be coupled into the fiber [Fig. 3(b)]. Temporal pulse shapes were continually monitored using an InGas amplified photodetector (PDA10D2, Thorlabs, Newton, New Jersey) connected to an oscilloscope (Tekronix, Beaverton, Oregon). If the spike was not eliminated for a pulse, that pulse was not used for data analysis, and the number of pulses in the train was increased until 20 spikeless pulses were delivered to the nerve.

\subsection{Data Analysis}

The stimulation threshold $\left(H_{50}\right)$ is defined as the radiant exposure at which $50 \%$ of the laser pulses evoked CMAP responses, and this definition is used to compare all data. To determine the stimulation threshold, recordings from each trial were used to determine the number of INSevoked CMAPs. It is important to note that CMAP amplitude increases with the magnitude of radiant exposure. ${ }^{20,62}$ For the purposes of this study, the presence of a CMAP regardless of its amplitude is used as the physiological end point to indicate successful INS, and thus the probability of evoking a CMAP is unaffected. Responses with a peak magnitude greater than two standard deviations above the baseline were considered evoked CMAPs. The number of evoked CMAPs was then divided by the total number of delivered pulses to determine the activation probability for every radiant exposure. A cumulative distribution function (CDF) of the standard normal distribution

$$
F\left(x ; \mu, \sigma^{2}\right)=\frac{1}{2}\left[1+\operatorname{erf}\left(\frac{x-\mu}{\sigma \sqrt{2}}\right)\right], \quad x \in \mathcal{R},
$$

where $x$ is the radiant exposure with mean $\mu$ and variance $\sigma^{2}$, was then fit to the data to determine the radiant exposure corresponding to $\mu$ or the $50 \%$ probability of evoking a CMAP $\left(H_{50}\right){ }^{63}$ While the $H_{50}$ is of little use for practical applications, this method is a common means to determine and compare dosimetric thresholds for radiation bioeffects. ${ }^{63-68}$ INS parameters with a lower $H_{50}$ are considered more efficacious as they require less energy and produce smaller temperature rises.

Another measure of stimulation efficacy is the transition rate to $100 \%$ activation probability. The transition rate to $100 \%$ activation probability is defined as the peak slope of the fitted CDF $\left(m_{\text {peak }}\right)$

$$
m_{\text {peak }}=\max \left[F^{\prime}\left(x ; \mu, \sigma^{2}\right)\right], \quad x \in \mathcal{R},
$$

where $F^{\prime}$ denotes the first derivative of $F$. This calculation represents the determinacy of the stimulation threshold. A larger $m_{\text {peak }}$ (i.e., a sharper transition rate) translates to a more reliable and predictable stimulation. This characteristic is more desirable since a smaller range of radiant exposures exists where one may experience ambiguity as to whether a given pulse evokes an action potential or not. Graphical representations of $H_{50}$ and $m_{\text {peak }}$ calculations can be found in Fig. S2 in the Supplemental Materials.

\subsection{Histological Evaluation}

Twelve rats were prepared using the surgical protocol described previously to expose both sciatic nerves. Five different sites on each nerve were irradiated under five different experimental conditions using a bare polished fiber at a spot size of $500 \mu \mathrm{m}$ : three experimental spots, a negative control spot, and a positive damage control spot. All five irradiation sites were spaced $1 \mathrm{~mm}$ apart and distributed axially along the nerve. All conditions were identical to the experimental protocol described above: a 10 -s pulse train at a repetition rate of $2 \mathrm{~Hz}$. The three experimental spots for each nerve were assessed at one, two, and three times the $H_{50}$. Radiant exposures of $\sim 6.0 \mathrm{~J} / \mathrm{cm}^{2}$ served as the positive damage control. The negative control was applied by positioning the fiber similarly to other trials while omitting irradiation. These experimental conditions were evaluated for damage with the $1450 \mathrm{~nm}$ and $1875 \mathrm{~nm}$ laser systems. Each irradiation site was marked with histological tissue dye (FisherBrand, Leicestershire, United Kingdom) for 
excision and histological preparation. After stimulation, nerves were immediately excised and placed in $4 \%$ paraformaldehyde for $48 \mathrm{~h}$. Fixed samples were then paraffin-embedded, sliced, and stained with toluidine blue to assess myelin damage. Histological slices were imaged under $60 \times$ magnification and examined for evidence of disruption and vacuolization of the myelin sheath, disruption of axons, ablation crater formation, and charring. ${ }^{57}$ Previous studies have rigorously determined the damage threshold for the Ho:YAG laser using the same criteria. ${ }^{57}$

\subsection{Statistical Analysis}

All statistical testing consisted of an ANOVA followed by a multiple comparison test using the Bonferroni method to account for small sample sizes.

\section{Results}

\subsection{Spot Size}

For each of the wavelengths, the $H_{50}$ remained consistent across all spot sizes (Fig. 4). The laser diode systems at $1450 \mathrm{~nm}$ and $1875 \mathrm{~nm}$ have a combined average $H_{50}$ of $1.01 \pm 0.06 \mathrm{~J} / \mathrm{cm}^{2}$ while the average $H_{50}$ for the Ho:YAG is $0.73 \pm 0.07 \mathrm{~J} / \mathrm{cm}^{2}$ across all spot sizes. No substantial difference between the stimulation efficacy of $1450 \mathrm{~nm}$ and $1875 \mathrm{~nm}$ diode lasers was observed ( $p>0.49$ for all comparisons). The $H_{50}$ for the Ho:YAG was lower than the diode lasers for every spot size. This difference was not significant $(p=0.29)$ for the $500 \mu \mathrm{m}$ spot size but was significant for the $800 \mu \mathrm{m}$ ( $p<0.02$ for $1450 \mathrm{~nm}$ and $1875 \mathrm{~nm}$ comparisons) and $1000 \mu \mathrm{m}$ spot sizes ( $p<0.014$ for $1450 \mathrm{~nm}$ and $1875 \mathrm{~nm}$ comparisons).

Note, diode lasers at $1875 \mathrm{~nm}$ tend to have less power output than their $1450 \mathrm{~nm}$ counterparts. Consequently, the radiant exposures at $500 \mu \mathrm{m}, 800 \mu \mathrm{m}$, and $1000 \mu \mathrm{m}$ spot sizes for the $1875 \mathrm{~nm}$ diode were achieved using $2 \mathrm{~ms}, 3 \mathrm{~ms}$, and $5 \mathrm{~ms}$ pulses, respectively. Despite the change in pulse width, however, the $H_{50}$ for $1875 \mathrm{~nm}$ was unaltered for all spot sizes.

\subsection{Pulse Width}

The effects of pulse width on $H_{50}$ were examined since the Ho:YAG produced a noticeably lower $H_{50}$ with $350 \mu$ s pulses compared to the $\geq 2 \mathrm{~ms}$ pulses from the diode lasers (Fig. 5). To compare pulse widths, a $1470 \mathrm{~nm}$ diode laser with the power specifications to produce adequate radiant exposures at $350 \mu \mathrm{s}$ pulses was used to investigate the role of pulse duration on INS efficacy. In addition, the absorption coefficient of water at $1470 \mathrm{~nm}\left(\mu_{a} \approx 24.9 \mathrm{~cm}^{-1}\right)$ is also more similar to the water absorption coefficient at $2120 \mathrm{~nm}\left(\mu_{a} \approx 24 \mathrm{~cm}^{-1}\right)$ than at either $1450 \mathrm{~nm}$ or $1875 \mathrm{~nm}$ (Table 1). At a spot size of $500 \mu \mathrm{m}$, the $H_{50}$ did not change significantly between pulse widths of

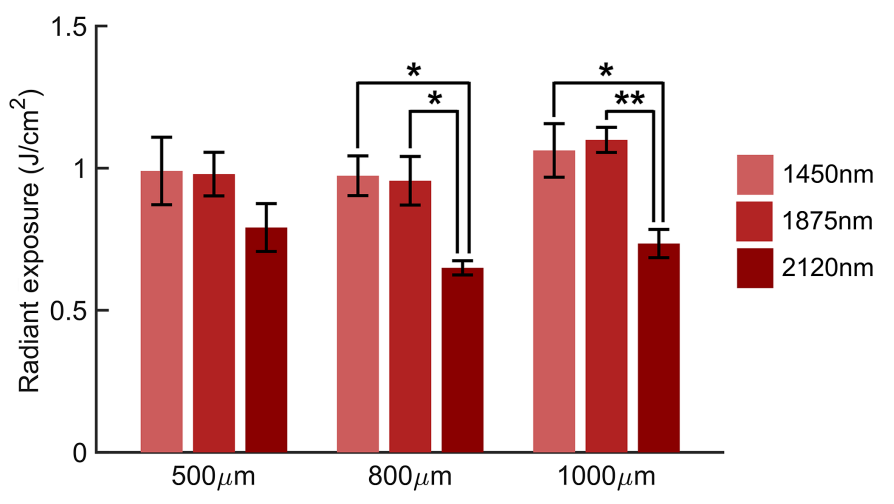

Fig. 4 Stimulation threshold remains constant across every spot size for a given wavelength while the Ho:YAG consistently produces a $H_{50}$ lower than that of both laser diode systems. $\left(H_{50} \pm \mathrm{SEM}, n=5\right)$. 


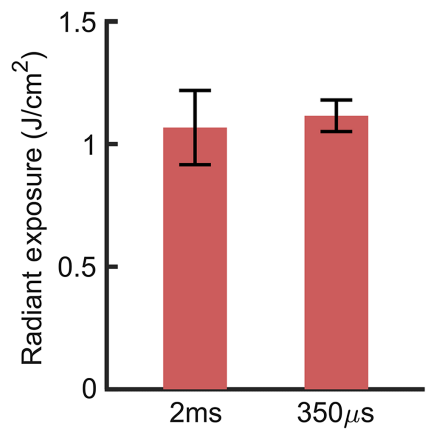

(a)

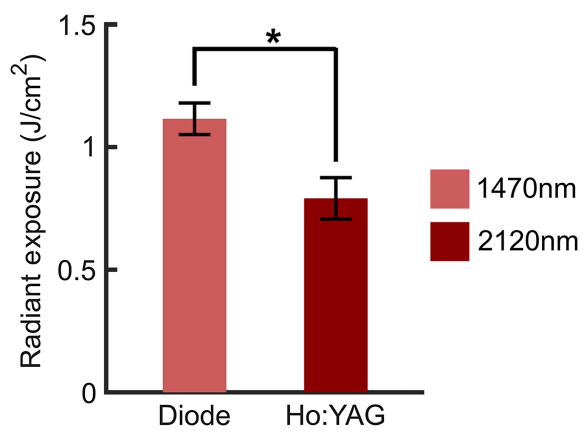

(b)

Fig. 5 Effects of pulse width on $H_{50}$. (a) Pulse width does not significantly alter the $H_{50}$ for diode lasers $\left(H_{50} \pm\right.$ SEM, $n=5,500 \mu \mathrm{m}$ spot size). (b) $H_{50}$ of the Ho:YAG laser is lower than that of the diode lasers at equal pulse widths $\left(H_{50} \pm \mathrm{SEM}, n=5, \tau_{p}=350 \mu \mathrm{s}, 500 \mu \mathrm{m}\right.$ spot size $)$.

$2 \mathrm{~ms}$ and $350 \mu$ s [Fig. 5(a), $p=0.38$ ]. Further, shortening of the pulse width did not account for the reduced $H_{50}$ of the Ho:YAG [Fig. 5(b)]. Despite having equal spot sizes and pulse widths, the $H_{50}$ of the Ho:YAG remained significantly lower than that of the $1470 \mathrm{~nm}$ laser diode $(p=0.016)$.

\subsection{Transitions in Activation Probability of Diode versus Ho:YAG Lasers}

The change in activation probability resulting from the various laser systems was further evaluated by comparing the CDFs of activation probability (Fig. 6). Combined CDFs based on all data collected for a defined experimental condition are depicted in Fig. $6\left(\right.$ a) $\left(\lambda_{5} \mathrm{~ms}=1875 \mathrm{~nm}\right.$, Spot Size $_{5 \mathrm{~ms}}=1000 \mu \mathrm{m} ; \quad \lambda_{2 \mathrm{~ms}}=1470 \mathrm{~nm}, \quad$ Spot Size $_{2 \mathrm{~ms}}=500 \mu \mathrm{m} ; \quad \lambda_{350 \mu \mathrm{s}}=1470 \mathrm{~nm}$, $\lambda_{2 \mathrm{~ms}}=1470 \mathrm{~nm}$, Spot Size $_{350 \mu \mathrm{s}}=500 \mu \mathrm{m} ; \lambda_{\mathrm{Ho}: \mathrm{YAG}}=2120 \mathrm{~nm}$, Spot Size $\left._{\mathrm{Ho}: \mathrm{YAG}}=500 \mu \mathrm{m}\right)$. Note that the $5 \mathrm{~ms}$ results are based on experiments using $1875 \mathrm{~nm}$ diode laser. Since pulse duration did not affect the $H_{50}$ as seen in Fig. 5, this is unlikely to affect the results presented here. The combined CDFs of the laser diode systems at $5 \mathrm{~ms}, 2 \mathrm{~ms}$, and $350 \mu$ s remain relatively broad and gradually transition to an activation probability of 1 . The distribution of the combined CDF for the Ho:YAG, however, is noticeably narrow with a steeper transition from an activation probability of 0 to 1 . This steep transition is reflected in the $m_{\text {peak }}$ for each laser and pulse width.

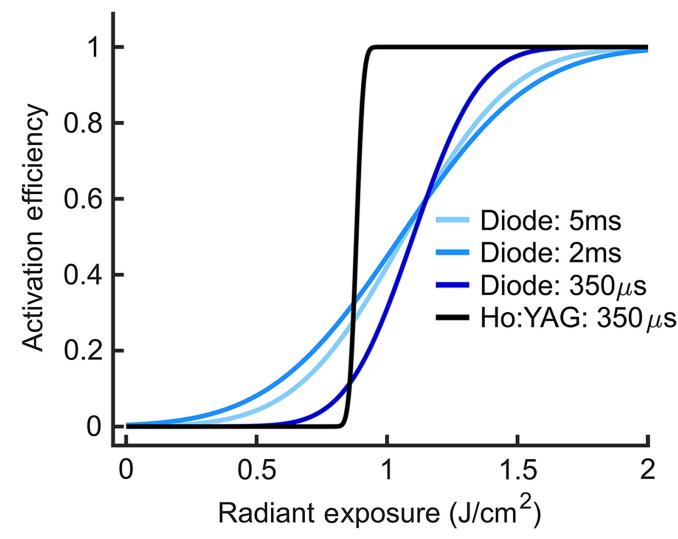

(a)

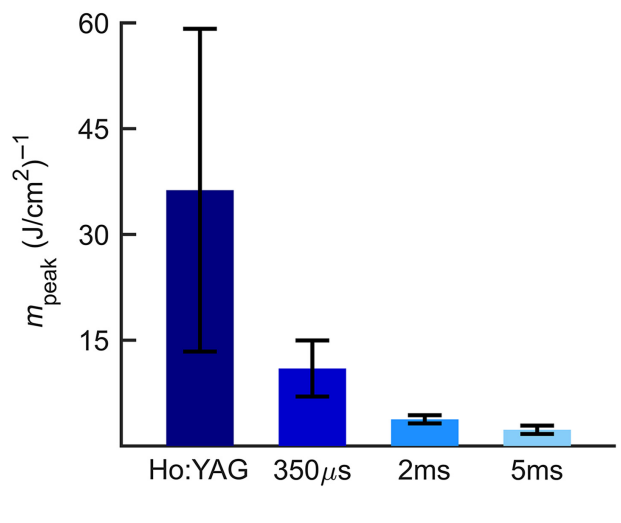

(b)

Fig. 6 Difference in activation probabilities between diode and Ho:YAG lasers. (a) CDFs fitted to all samples from diode and Ho:YAG lasers for a given set of parameters. $\left(\lambda_{5} \mathrm{~ms}=1875 \mathrm{~nm}\right.$, Spot Size ${ }_{5 \mathrm{~ms}}=1000 \mu \mathrm{m} ; \lambda_{2 \mathrm{~ms}}=1470 \mathrm{~nm}$, Spot Size $2 \mathrm{~ms}=500 \mu \mathrm{m} ; \lambda_{350 \mu \mathrm{s}}=1470 \mathrm{~nm}, \lambda_{2 \mathrm{~ms}}=$ $1470 \mathrm{~nm}$, Spot Size $350 \mu \mathrm{s}=500 \mu \mathrm{m} ; \lambda_{\mathrm{Ho}}: \mathrm{YAG}=2120 \mathrm{~nm}$, Spot Size $\left.\mathrm{Ho}_{\mathrm{YAG}}=500 \mu \mathrm{m}\right)$. (b) The transition rate ( $\left.m_{\text {peak }}\right)$ of diode lasers at various pulse widths and Ho:YAG laser at $350 \mu$ s pulse width $\left(m_{\text {peak }} \pm \mathrm{SEM}, n=5\right)$. A larger $m_{\text {peak }}$ corresponds to a steeper transition in activation probability. 
The transition to an activation probability of 1 can be quantified by the peak slope of the $\mathrm{CDF}, m_{\text {peak }}$. A larger $m_{\text {peak }}$ implies that there is a steeper transition from an activation probability of 0 to 1 that occurs quickly over a smaller range of radiant exposures than an activation CDF with a smaller $m_{\text {peak }}$. In comparison to the diode lasers, the $m_{\text {peak }}$ of the Ho:YAG is considerably higher [Fig. 6(b)]. The $m_{\text {peak }}$ of the diodes increases with decreasing pulse width while the $m_{\text {peak }}$ of the Ho:YAG is greater than that of the $1470 \mathrm{~nm}$ diode laser at the same pulse width.

\subsection{Effects of Ho:YAG Pulse Shape on Stimulation Efficacy}

In light of the lower $H_{50}$ and greater $m_{\text {peak }}$ of the Ho:YAG laser at equivalent pulse widths and radiant exposures to the diode lasers, the effect of the temporal pulse shape of the Ho:YAG

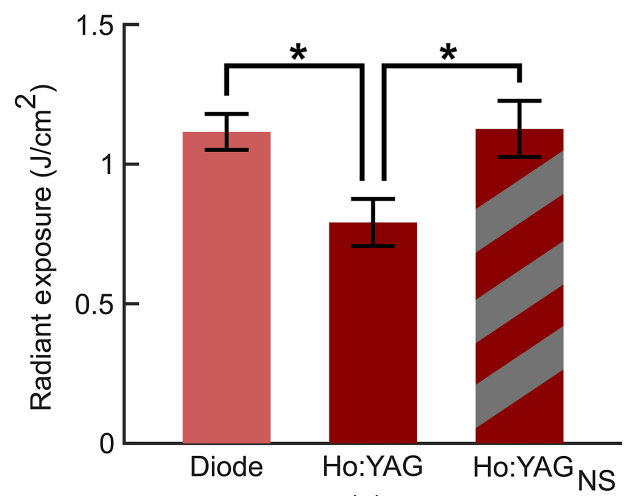

(a)

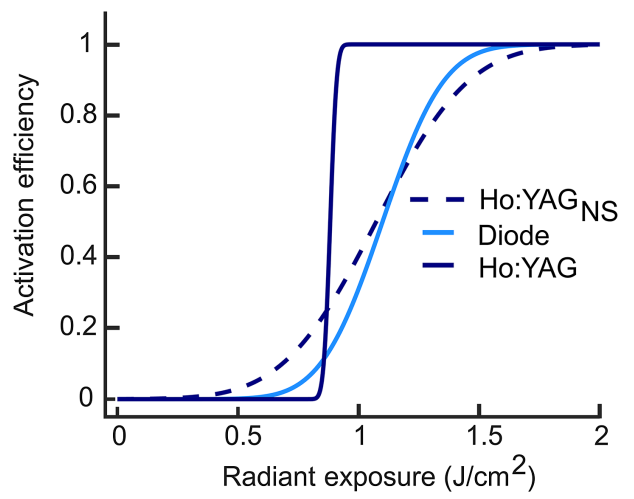

(b)

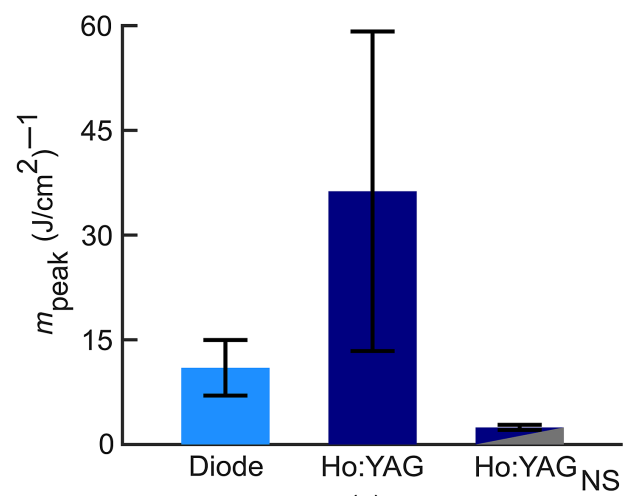

(c)

Fig. 7 The effects of spikeless Ho:YAG pulse on stimulation efficacy. (a) Spikeless pulses produced a greater $H_{50}$ than the unaltered $\mathrm{Ho}: Y A G$ pulse $\left(\mathrm{H}_{50} \pm \mathrm{SEM}, n=5\right)(\mathrm{b})$ Fitted CDFs to all data for the spikeless Ho:YAG, unaltered Ho:YAG, and diode lasers. (c) The transition rate $\left(m_{\text {peak }}\right)$ of the spikeless Ho:YAG, unaltered Ho:YAG, and diode lasers $\left(m_{\text {peak }} \pm \mathrm{SEM}, n=5\right)$. For all experiments: spot size $=500 \mu \mathrm{m}, \lambda_{\text {Diode }}=1470 \mathrm{~nm}, \tau_{p}=350 \mu \mathrm{s}$. NS, spikeless. 
was investigated. In comparing the pulse shapes of the two lasers, the most distinct feature of the asymmetric Ho:YAG pulse is the microsecond spike at the onset of the pulse [Fig. 3(a)] which is characteristic of flashlamp-pumped Ho:YAG lasers. The microsecond spike has a peak intensity that is $\sim 5 \times$ greater than that of the diode laser and thus a greater peak power. To determine whether the spike played a role in the stimulation efficacy of the Ho:YAG laser, the spike was removed from the pulse and experiments repeated with spikeless pulses (see Sec. 2.3.3).

The spikeless Ho:YAG pulse produced a significantly higher $H_{50}$ than the unaltered Ho:YAG pulse [ $p=0.025$; Fig. 7(a)]. Moreover, the $H_{50}$ of the spikeless pulses did not differ compared to the $H_{50}$ of $1470 \mathrm{~nm}$ at the same $350 \mu$ s pulse width and same $500 \mu \mathrm{m}$ spot size $(p=1)$. The spikeless Ho:YAG pulse also yielded notable differences in activation probability. The $m_{\text {peak }}$ of the spikeless Ho:YAG pulses was remarkably less than that of the normal Ho:YAG pulses [Fig. 7(c)]. The $m_{\text {peak }}$ of the spikeless pulses was similar to the diode laser at an equal pulse width. The change in $m_{\text {peak }}$ of the spikeless Ho:YAG pulse to mirror that of the diode pulse is visually apparent in the CDFs fitted to each dataset [Fig. 7(b)].

\subsection{Histological Damage Assessment}

Histological safety was evaluated by assessing myelin integrity using a toluidine blue stain. No signs of damage were observed in the negative control [Fig. 8(a)]. Clear evidence of charring and hyalinization were observed in the positive control at $\sim 6 \mathrm{~J} / \mathrm{cm}^{2}[$ Fig. 8(b)]. Neither disruption of myelin sheath nor charring was observed for 1875 or $1450 \mathrm{~nm}$ even at three times the $H_{50}$ threshold [3 J/ $\mathrm{cm}^{2}$; Figs. 8(c) and 8(d)]. Results were consistent across all samples irradiated at one, two, and three times the $H_{50}$ radiant exposure. These results imply that INS with both 1875 and $1450 \mathrm{~nm}$ have a safety ratio of $\sim 3: 1$.

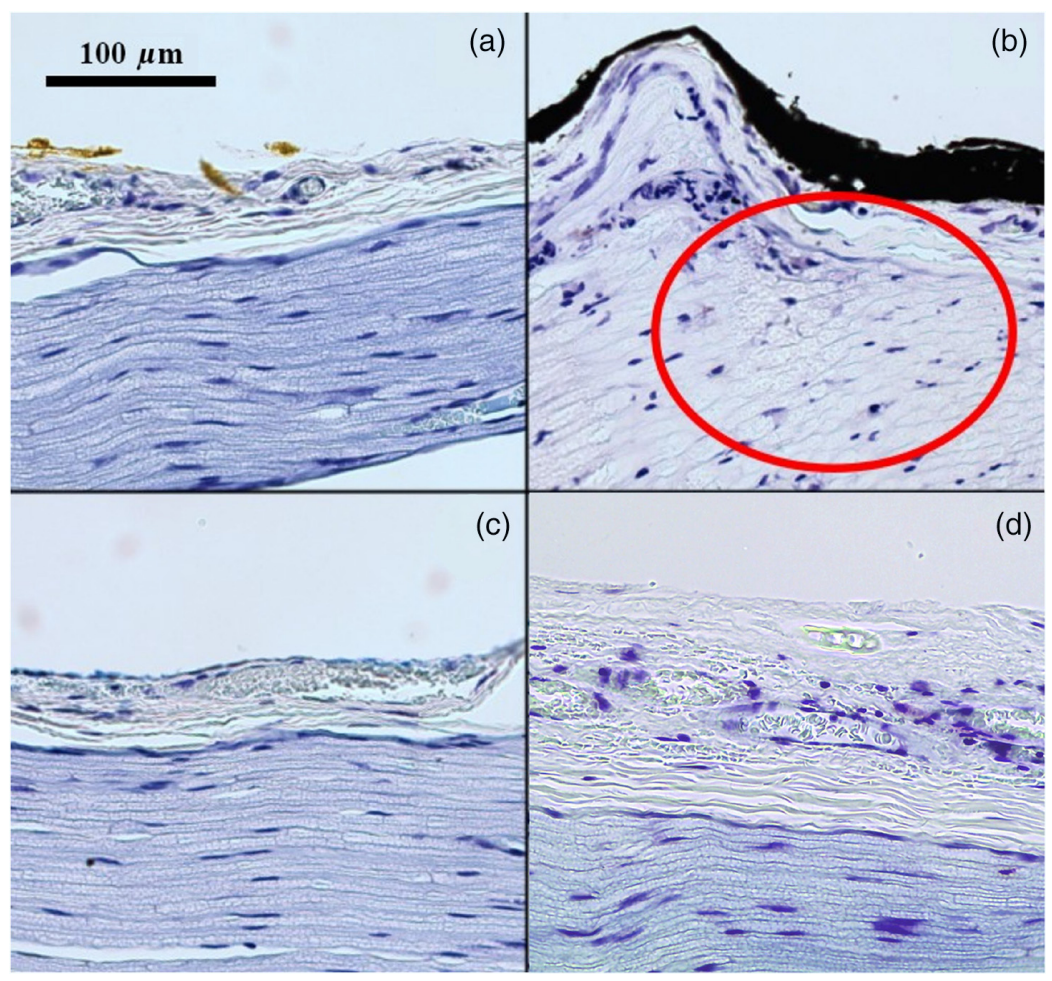

Fig. 8 Representative irradiated nerve slices stained with toluidine blue. (a) Negative control, no stimulation. (b) Positive control: $6 \mathrm{~J} / \mathrm{cm}^{2}$ at $1450 \mathrm{~nm}$ light. Red ellipse indicates region of myelin disruption. (c) $3.08 \mathrm{~J} / \mathrm{cm}^{2}$ irradiation at $1450 \mathrm{~nm}$. (d) $3.15 \mathrm{~J} / \mathrm{cm}^{2}$ irradiation at $1875 \mathrm{~nm}$. 


\section{Discussion}

Optical neuromodulation has had a profound impact on both clinical and basic research due to its spatial selectivity. In clinical applications where high spatial resolution is required including prostatectomies, ${ }^{16,33,34}$ rhizotomies, ${ }^{29}$ and skull base surgeries, ${ }^{35}$ optical methods could offer a superior alternative to electrical stimulation. With optogenetics, selective neural excitation requires targeting genetically altered neurons. This genetic specificity, however, presents translational difficulties in humans due to unknown immune reactivity and potential ethical constraints. INS circumvents these limitations by offering high spatial selectivity without the need for genetic modification or exogenous substrates. For these reasons, INS is a particularly well-suited method for clinical translation. Hence, in this study, we determined the optimal parameters for INS efficacy while confirming the identified parameters did not risk damage. To that end, the results of this study show shorter pulse durations improve activation probability and diode lasers exhibit a $\sim 3: 1$ safety ratio regardless of spot size.

Both the $1450 \mathrm{~nm}$ and $1875 \mathrm{~nm}$ diode lasers have a consistent $H_{50}$ of $\sim 1 \mathrm{~J} / \mathrm{cm}^{2}$ across all considered spot sizes (Fig. 4). Thus, in broadening the spot size, the primary compromise is sacrificing the intrinsic spatial selectivity of INS. Since water serves as the primary chromophore in the SWIR responsible for the heat generation needed for INS, we initially expected $1450 \mathrm{~nm}$, which has a higher water absorption coefficient than $1875 \mathrm{~nm}$, to have a lower $H_{50}$. Moreover, scattering is considered negligible in the SWIR as absorption coefficients are considerably higher than the effective scattering. ${ }^{18}$ However, when the source term, $S(z)$, given by

$$
S(z)=\mu_{a} H_{0} e^{-\mu_{a} z},
$$

which describes the absorbed energy density at a given depth in $\mathrm{J} / \mathrm{cm}^{3}$ where $H_{0}$ is the initial radiant exposure and $z$ is the depth of laser penetration in tissue, is compared for the two wavelengths along with the anatomy of the rat sciatic nerve, ${ }^{69}$ the deposited energy from each wavelength within the nerve fascicle itself is nearly equivalent (Fig. S3 in the Supplemental Materials). As the absorbed energy is the driving force underlying heat generation and thus INS, this comparison is of more consequence than the relative absorption coefficients. In addition, the endpoint used to determine successful stimulation is a CMAP which is a secondary and collective response from the population of excited axons. Thus, CMAPs are not dependent on the activation of a single axon at a specific depth but rather the activation of multiple axons over the entire irradiated volume. The similarity in $H_{50}$ of the three diode lasers used here is likely due to the fact that a similar amount of energy is deposited within the nerve fascicles themselves. The $H_{50}$ of the Ho: YAG laser, however, is lower than that of the diode lasers at $\sim 0.7 \mathrm{~J} / \mathrm{cm}^{2}$, which is consistent for every spot size. For the $800 \mu \mathrm{m}$ and $1000 \mu \mathrm{m}$ spot sizes, this difference between the $H_{50}$ of the diodes and Ho:YAG lasers is statistically significant ( $p<0.02$ for all comparisons). This difference, at first glance however, seems to contradict the initial observations of Wells et al. ${ }^{18}$

Wells et al. did not observe the same difference in the stimulation threshold between diode lasers and Ho:YAG lasers in the same in vivo rat sciatic nerve model. ${ }^{18}$ Rather the stimulation threshold was consistently between 0.4 and $0.5 \mathrm{~J} / \mathrm{cm}^{2}$ for both lasers (see Fig. 2 in Ref. 18). The difference in reported values, however, is due to a difference in nomenclature and radiant exposure calculation. Here, the stimulation threshold refers to the $H_{50}$ or the radiant exposure at which $50 \%$ of the pulse will evoke a CMAP, whereas Wells et al. defined the stimulation threshold as the lowest radiant exposure to evoke a CMAP. Hence, the $H_{50}$ is expected to be greater than the stimulation threshold values reported by Wells et al. In adopting the stimulation threshold definition and spot size calculation of Wells et al., our data yield similar results with a stimulation threshold of $0.32 \mathrm{~J} / \mathrm{cm}$ and $0.39 \mathrm{~J} / \mathrm{cm}$ for the Ho:YAG laser and $1875 \mathrm{~nm}$ diode laser with $2 \mathrm{~ms}$ pulses, respectively. Moreover, whereas the spot sizes measured here were determined using the knife-edge technique, Wells et al. calculated their values based on the fiber diameter, the distance from the fiber to tissue, and the numerical aperture of the fiber. As the radiant exposure is inversely proportional to the square of the beam radius, the difference in calculated versus measured values also likely accounts for any discrepancies in the reported values. Nonetheless, seeing the evident difference between the $H_{50}$ of the diode lasers and the Ho:YAG observed here, the influence of pulse width on the efficacy of INS was then investigated. 
While the pulse width of the Ho:YAG is fixed at $350 \mu$ s, the diode lasers have an adjustable pulse width. To create the necessary range of radiant exposures for the CDFs at each spot size in Fig. 4, the pulse widths of the $1875 \mathrm{~nm}$ diode laser were modulated. For the $500 \mu \mathrm{m}, 800 \mu \mathrm{m}$, and $1000 \mu \mathrm{m}$ spot sizes, the pulses were $2 \mathrm{~ms}, 3 \mathrm{~ms}$, and $5 \mathrm{~ms}$, respectively. Previously, Wells et al. also showed that the pulse width does not alter the stimulation threshold for INS with a constant spot size. ${ }^{18}$ Here, Fig. 4 similarly shows that even with changes in spot size and pulse width, the $H_{50}$ is unaffected for a given laser type $(p=0.34)$. To confirm that the improved efficacy of the Ho:YAG is not due to its shorter pulse width, the $H_{50}$ of the Ho:YAG is compared to a $1470 \mathrm{~nm}$ diode laser capable of generating equal radiant exposures at a $350 \mu$ s pulse width. The data reaffirm that a pulse width of $350 \mu$ s versus $2 \mathrm{~ms}$ offers no advantage in terms of lowering the $H_{50}$ with the $1470 \mathrm{~nm}$ diode laser [Fig. 5(a)]. In Fig. 5(b), the $H_{50}$ for the Ho: YAG remains lower than the $H_{50}$ of the $1470 \mathrm{~nm}$ even with an equal pulse width, spot size, and radiant exposures. In addition to both lasers generating comparable optical energy for INS, $1470 \mathrm{~nm}$ light also has an almost equivalent water absorption coefficient to $2120 \mathrm{~nm}$ (Table 1). Consequently, this makes it unlikely that any observed differences are due to changes in optical absorption, penetration depth, or heat generation within the tissue. Taken together, the shorter pulse width of the Ho:YAG is not responsible for its lower $H_{50}$.

Though the $H_{50}$ across all lasers are unaffected by pulse width and spot size, the transition in activation probability (i.e., $m_{\text {peak }}$ ) increases as the pulse width of the diode lasers decreases (Fig. 6). Figure 6(a) depicts this observation graphically by plotting the CDF fitted to the combined data points from all trials of identical experimental conditions. As the pulse width decreases from $5 \mathrm{~ms}$ to $350 \mu \mathrm{s}$, the CDFs corresponding to the diode lasers transition to an activation probability of 1 more sharply over a smaller range of radiant exposures (i.e., have a steeper slope $\left(m_{\text {peak }}\right)$. In other words, the response becomes more binary as the pulse width gets shorter, and a stimulation threshold is more readily identifiable. The rate of this transition is represented by the $m_{\text {peak }}$ of each CDF [Fig. 6(b)]. The smaller $m_{\text {peak }}$ of shorter pulse widths suggests that shorter pulse widths more consistently satisfy the conditions for the thermal gradient needed to generate an action potential. This observation complements the previous findings of Shapiro et al., who showed that for pulses $\leq 2 \mathrm{~ms}$, the evoked inward current was shorter in duration and greater in amplitude than $10 \mathrm{~ms}$ pulses at $1889 \mathrm{~nm} .{ }^{22}$ Moreover, the evoked currents for lower energy $(1.4 \mathrm{~mJ}$ and $2.8 \mathrm{~mJ})$ pulses $\leq 2 \mathrm{~ms}$ were greater despite the higher energy $(7.3 \mathrm{~mJ})$ of the $10 \mathrm{~ms}$ pulse. Inward currents resulting from a 10-ms pulse were substantially longer in duration and mirrored the square shape of the pulse, suggesting that pulse width plays a crucial role in the duration and amplitude of the evoked depolarizing currents. Because INSinduced inward currents are responsible for action potential initiation, the shorter duration and larger magnitude inward currents elicited from pulse widths $\leq 2 \mathrm{~ms}$ may explain why diode lasers more reliably excite CMAPs at shorter pulse widths and why the $m_{\text {peak }}$ is smaller for the $5 \mathrm{~ms}$ pulses examined here [as shown in Fig. 6(b)]. The reliability of shorter pulse durations could also have a thermodynamic explanation. The shorter pulse durations deposit infrared light within the tissue more quickly, allowing less time for thermal diffusion and potentially allowing a larger portion of the optical energy to directly generate the thermal gradient. However, even at an equal pulse width, the Ho:YAG consistently exhibited a much sharper transition in activation probability than the diode lasers.

The Ho:YAG generates an almost "all-or-none" response in which either every pulse will evoke a CMAP or none will. Figure 6(a) shows this feature of the Ho:YAG where the CDF resembles a step function. Further, the Ho:YAG has a greater $m_{\text {peak }}$ than the diode laser at an equal pulse width [Fig. 6(b)]. The sharper transitions of the Ho:YAG corresponds to more reliable stimulation (activation probability of 1 ) than the diode systems when only slightly above the $H_{50}$ and removes any ambiguity in achieving stimulation. This observation has clear clinical and experimental implications when using the Ho:YAG laser; INS is more likely to have an activation probability of 1 at a lower radiant exposure than the diode lasers. Though not on par with Ho:YAG laser, the higher power output of diode lasers centered near $1450 \mathrm{~nm}$ is a clear advantage over those centered near $1875 \mathrm{~nm}$. Diode lasers at $1450 \mathrm{~nm}$ generally have a greater power output than $1875 \mathrm{~nm}$ diode lasers enabling them to produce the necessary radiant exposures needed at shorter pulse widths. Consequently, diode lasers centered near $1450 \mathrm{~nm}$ can achieve a higher $m_{\text {peak }}$ and sharper transitions in activation probability than $1875 \mathrm{~nm}$ diodes. 
Changing the pulse width of the $1450 \mathrm{~nm}$ diode laser, however, did not fully account for the larger $m_{\text {peak }}$ seen when using the Ho:YAG laser as compared the $1450 \mathrm{~nm}$ diode laser.

Figure 3(a) shows typical $350 \mu$ s pulse shapes from the $1470 \mathrm{~nm}$ diode and Ho:YAG lasers. Both pulses contain equal integrated areas and thus would deliver equal energies per pulse. The trace from the diode laser resembles a uniform square pulse mirroring the driving current. Conversely, the Ho:YAG pulse has an asymmetric shape with a $\sim 1 \mu$ s spike at the onset of the pulse. This pulse shape is a result of the Ho:YAG being pumped by a flashlamp as compared to a driving current. As the spike produces a higher peak power, its contribution to the stimulation efficacy of the Ho:YAG was investigated. Unfortunately, due to the rise-time of the diode, these lasers cannot generate similar pulse shapes. Instead, the effect of removing the spike in the Ho: YAG pulse was investigated. Experiments using spikeless Ho:YAG pulses resulted in a $H_{50}$ nearly equivalent to the $350 \mu$ s pulses of the $1470 \mathrm{~nm}$ diode laser [Fig. 7(a)]. Moreover, the $H_{50}$ from the spikeless pulse resulted in a significantly greater $H_{50}$ compared to the unaltered Ho:YAG pulses. The elimination of the microsecond spike also lowered the $m_{\text {peak }}$ of the Ho:YAG and once again produced results more similar to the diode laser. Consequently, both the spikeless Ho:YAG and diode laser pulses have a notably lower $m_{\text {peak }}$ than the unaltered Ho:YAG pulses [Fig. 7(c)]. Taken together, the results suggest that the microsecond spike at the onset of Ho:YAG pulses is largely responsible for the superior stimulation efficacy of the Ho:YAG laser compared to the diode lasers.

Based on the data, the lower $H_{50}$ and higher $m_{\text {peak }}$ of the Ho:YAG laser may be due to the rapid deposition of energy from the initial high intensity spike of the pulse. This finding aligns well with previous studies by Izzo et al.$^{70,71}$ Using a $1937 \mathrm{~nm}$ diode laser, Izzo et al. demonstrated that the stimulation threshold to elicit a CAP in the gerbil cochlea decreases with decreasing pulse widths. ${ }^{71}$ Interestingly, they also noted a significant decrease in the stimulation threshold going from $300 \mu \mathrm{s}$ to $100 \mu$ s pulses that gradually continued to decrease down to $5 \mu$ s pulse durations. In another study, Izzo et al. saw the same trend using a $1860 \mathrm{~nm}$ diode laser again in the gerbil cochlea this time noting that the peak power at the stimulation thresholds was constant from 100 to $1000 \mu$ s pulses but increased for pulse widths $\leq 35 \mu \mathrm{s} .^{70}$ The Ho:YAG pulse, with its leading microsecond spike, may elicit a similar effect as observed by Izzo et al., creating a higher peak power and therefore lowering the stimulation threshold as seen at pulse durations $<100 \mu \mathrm{s}$. Again, this effect could be the result of higher peak powers more consistently generating the thermal gradient needed for INS. Moreover, based on their data, Izzo et al. suggested that the thermal relaxation time for their optical target is in the range of $\sim 30$ to $100 \mu \mathrm{s}^{71}$ Thus, with shorter pulse widths $(\leq 30 \mu \mathrm{s})$ like the microsecond spike in the Ho:YAG pulse, the optical energy contained within the pulse is deposited within the nerve creating the required thermal rise well before the heat has time to dissipate (i.e., the pulse width is less than thermal relaxation time constant of the tissue). Given the controversy surrounding the mechanism of INS in the auditory system, ${ }^{25}$ it should be noted that Izzo et al. did not use a deafened animal model, and an opto-acoustic or thermal expansion model is consistent with the minimum power required for intracochlear stimulation, even though the pulse durations used do not meet conditions of stress confinement at this wavelength. ${ }^{72}$

These observations also complement the research done with photoabsorber-induced neurothermal stimulation (PAINTS). ${ }^{73,74}$ Similar to INS, PAINTS utilize exogenous chromophore absorption to achieve photothermal neural stimulation in order to provide more flexibility in targeting deeper neural structures. In cultured rat brain slices, Farah et al. demonstrated that the stimulation threshold for rat brain slice cultures decreases with pulse width and plateaus around $50 \mu$ s while using a $800 \mathrm{~nm}$ titanium-sapphire laser. ${ }^{73}$ Furthermore, pure thermal stimulation threshold curves failed to predict the results obtained from these short stimulation pulses suggesting an auxiliary nonthermal mechanism. Farah et al. hypothesize that the auxiliary mechanism may be photomechanical in nature as microsecond pulses are more likely to induce pressure wave transients than longer millisecond pulses. The data presented in this current study appear to lend credence to this hypothesis.

As stated previously, Shapiro et al. demonstrated that lower energy pulses $\leq 2 \mathrm{~ms}$ evoked inward currents shorter in duration and higher in amplitude than higher energy $10 \mathrm{~ms}$ pulses. ${ }^{22}$ Given the data presented here and assuming this trend holds, there is substantial evidence that the initial microsecond spike of the Ho:YAG pulse may be responsible for the lower 
$H_{50}$ and sharper transitions in the activation probability of the Ho:YAG laser stimulation. Moreover, if the spike is considered independently from the rest of the pulse, a microsecond pulse is much closer to the stress confinement regime than the other pulse durations considered here. Though no empirical evidence is offered here, weak photomechanical effects may exist during Ho:YAG stimulation as photomechanical and photothermal effects are not easily decoupled. Therefore, Ho:YAG stimulation could possibly initiate action potentials through the same universal INS mechanism proposed by Plaksin, Shoham, and Kimmel, via a partially photomechanically driven rather than a solely photothermally driven capacitance change. ${ }^{23}$ The reader should note, however, that data presented here and that by Shapiro et al. use substantially different experimental setups.

In translating INS for clinical applications, the data from the standard and spikeless Ho: YAG experiments suggest that brief low-energy pulses with durations on the order of a microsecond may yield a more reliable approach for INS. The microsecond spike only contains $8 \%$ to $11 \%$ of the total pulse energy and yet has a profound effect on the stimulation efficacy. If INS only requires short, low-energy pulses, then applying this technique becomes highly advantageous for clinical applications as lower energies further reduce the risk of laser-induced damage. At the time of writing, however, the authors are unaware of any cost-effective laser source capable of producing the combination of low energy and short pulses at appropriate wavelengths. Though not investigated as part of this study, using a collimated beam also has practical, clinical implications. Radiant exposure is inversely proportional to the square of the beam radius incident on the tissue. With a bare optical fiber, the incident spot is dependent on the distance of the fiber output to the tissue. Thus, in handheld probes, slight changes in the distance of the fiber from tissue, as is common during clinical procedures, can drastically change the radiant exposure. A collimated beam provides a consistent spot size over a wide range of distances keeping the magnitude of the radiant exposure constant over multiple fiber placements or adjustments.

Lastly, we demonstrate the acute histological safety of $1450 \mathrm{~nm}$ light for INS for the first time (Fig. 8). Histology slides were examined using a binary grading scheme similar to previous studies where signs of damage included granular disruption and vacuolization of myelin sheaths of nerve fibers, disruption of axons, charring, and ablation crater formation. ${ }^{57}$ At radiant exposures three times their $H_{50}\left(\sim 3 \mathrm{~J} / \mathrm{cm}^{2}\right)$, the $1450 \mathrm{~nm}$ and $1875 \mathrm{~nm}$ diode lasers show no signs of myelin disruption or charring [Figs. 8(c) and 8(d)]. Myelin disruption was observed at radiation exposures $>3 \mathrm{~J} / \mathrm{cm}^{2}$. Thus, both diode lasers behave comparably with a shared safety ratio of $\sim 3: 1$. The safety ratio identified here is similar to the acute safety ratio observed with the Ho: YAG laser, which has been thoroughly characterized in previous studies. ${ }^{57}$ This validates the use of diode lasers at $1450 \mathrm{~nm}$ and $1875 \mathrm{~nm}$ for INS despite their higher $H_{50}$ and lower $m_{\text {peak }}$ values as compared to the more effective Ho:YAG laser.

Taken together, these findings confirm that INS can effectively and safely excite neural activity in the rat sciatic nerve. The data corroborate previous conclusions concerning the effects of pulse duration and stimulation threshold while also showing that $1450 \mathrm{~nm}$ laser diodes can serve as a more useful source for INS than the $1875 \mathrm{~nm}$ diode lasers.

Regardless of the advantages of the Ho:YAG laser, the SWIR diode laser remains an effective, low-cost, and user-friendly means of INS. The results presented here provide valuable insight into maximizing the efficacy of diode lasers for INS. While the $H_{50}$ of the diode lasers is constant regardless of pulse width, shortening the duration of the pulse width leads to more reliable stimulation due to a higher transition rate in activation probability. INS at shorter pulse widths is especially achievable when using laser diodes centered near $1450 \mathrm{~nm}$ as these diodes can generate sufficient radiant exposures at shorter pulse widths than $1875 \mathrm{~nm}$ diodes. The spot size plays little to no role in the efficacy of INS but comes at the cost of spatial selectivity. For the first time, histological evidence that $1450 \mathrm{~nm}$ and $1875 \mathrm{~nm}$ exhibit similar damage thresholds and safety ratios is presented. The diode lasers have a safety ratio of 3:1 compared to the 2:1 safety ratio of the Ho:YAG. ${ }^{57}$ Since we have shown that $1450 \mathrm{~nm}$ and $1875 \mathrm{~nm}$ diode lasers are practically equivalent laser sources for INS, the wider commercial availability of $1450 \mathrm{~nm}$ diode and its higher power output can be leveraged to improve the accessibility and versatility of INS in both experimental and clinical settings. 


\section{Conclusion}

We show that the $1450 \mathrm{~nm}$ diode lasers represent an effective and safe laser source for INS application on par with the more commonly used $1875 \mathrm{~nm}$ diode lasers. The Ho:YAG laser was shown to have greater stimulation efficacy than diode lasers primarily due to the initial spike at the onset of its pulse. Though the Ho:YAG laser outperformed the laser diode systems, diode lasers remain a cost-effective and reliable laser source for INS. These results also provide easy steps one can take to optimize the performance of diode lasers for INS. Looking forward, a compact, true pulsed laser would be the ideal laser system for clinical INS. The $1450 \mathrm{~nm}$ diode laser systems, however, provide an optimal compromise to explore INS strategies. Together, our results offer insight into further avenues for improving INS and its laser sources as a tool for label-free neural modulation.

\section{Disclosures}

The authors have declared they have no conflicts of interest.

\section{Acknowledgments}

This work was supported by the Vanderbilt Biophotonics Center and funded by the following grants: Air Force Office of Scientific Research (AFOSR) Defense University Research instrumentation Program (DURIP) FA9550-15-1-0328, AFOSR FA9550-14-1-0303, AFOSR FA9550-17-10374. GAT was supported through a National Science Foundation GRFP Fellowship. Authors wish to thank Dr. Manquing Wang and Dr. William Roach for their guidance in performing animal surgeries and data analysis.

\section{References}

1. G. Fritsch and E. Hitzig, "Über die elektrische Erregbarkeit des Grosshirns," Arch. Anat. Physiol. Wissen 37, 300-332 (1870).

2. L. A. Geddes and J. D. Bourland, "Tissue stimulation: theoretical considerations and practical applications," Med. Biol. Eng. Comput. 23, 131-137 (1985).

3. J. K. Song, B. Abou-Khalil, and P. E. Konrad, "Intraventricular monitoring for temporal lobe epilepsy: report on technique and initial results in eight patients," J. Neurol. Neurosurg. Psychiatry 74, 561-565 (2003).

4. R. Ishii et al., Neuromodulation in Basic, Translational and Clinical Research in Psychiatry, Lausanne Frontiers Media SA (2020).

5. J. Wells et al., "Application of infrared light for in vivo neural stimulation," J. Biomed. Opt. 10, 064003 (2005).

6. K. C. McGill et al., "On the nature and elimination of stimulus artifact in nerve signals evoked and recorded using surface electrodes," IEEE Trans. Biomed. Eng. BME-29, 129-137 (1982).

7. A. I. Matic, J. T. Walsh, and C.-P. Richter, "Spatial extent of cochlear infrared neural stimulation determined by tone-on-light masking," J. Biomed. Opt. 16, 118002 (2011).

8. A. G. Xu et al., "Focal infrared neural stimulation with high-field functional MRI: a rapid way to map mesoscale brain connectomes," Sci. Adv. 5, eaau7046 (2019).

9. A. W. Roe et al., "In vivo mapping of cortical columnar networks in the monkey with focal electrical and optical stimulation," Front. Neuroanat. 9, 135 (2015).

10. L. Wojtecki et al., "Pathological crying induced by deep brain stimulation," Mov. Disord. 22, 1314-1316 (2007).

11. P. Mahlknecht et al., "Pyramidal tract activation due to subthalamic deep brain stimulation in Parkinson's disease," Mov. Disord. 32, 1174-1182 (2017).

12. S. Pinto et al., "Stimulation of the pedunculopontine nucleus area in Parkinson's disease: effects on speech and intelligibility," Brain 137, 2759-2772 (2014).

13. D. Popovic et al., "Properties of implanted electrodes for functional electrical stimulation," Ann. Biomed. Eng. 19, 303-316 (1991). 
14. D. H. Liang and H. S. Lusted, "The nerve-electrode interface of the cochlear implant: current spread," IEEE Trans. Biomed. Eng. 46, 35-43 (1999).

15. R. L. Testerman, "Comments on 'accuracy limitations of chronaxie values," IEEE Trans. Biomed. Eng. 52, 750 (2005).

16. S. Tozburun et al., "Gaussian versus flat-top spatial beam profiles for optical stimulation of the prostate nerves," Proc. SPIE 7548, 75484W (2010).

17. J. W. Lee et al., "Emerging neural stimulation technologies for bladder dysfunctions," Int. Neurourol. J. 19, 3-11 (2015).

18. J. Wells et al., "Biophysical mechanisms of transient optical stimulation of peripheral nerve," Biophys. J. 93, 2567-2580 (2007).

19. J. Wells et al., "Optical stimulation of neural tissue in vivo," Opt. Lett. 30, 504-506 (2005).

20. J. Wells et al., "Pulsed laser versus electrical energy for peripheral nerve stimulation," J. Neurosci. Methods 163, 326-337 (2007).

21. E. S. Boyden et al., "Millisecond-timescale, genetically targeted optical control of neural activity," Nat. Neurosci. 8, 1263-1268 (2005).

22. M. G. Shapiro et al., "Infrared light excites cells by changing their electrical capacitance," Nat. Commun. 3, 736 (2012).

23. M. Plaksin et al., "Thermal transients excite neurons through universal intramembrane mechanoelectrical effects," Phys. Rev. X 8, 011043 (2018).

24. G. P. Tolstykh et al., "Ryanodine and IP 3 receptor-mediated calcium signaling play a pivotal role in neurological infrared laser modulation," Neurophotonics 4, 025001 (2017).

25. N. Kallweit et al., "Optoacoustic effect is responsible for laser-induced cochlear responses," Sci. Rep. 6, 28141 (2016).

26. H. T. Beier et al., "Plasma membrane nanoporation as a possible mechanism behind infrared excitation of cells," J. Neural Eng. 11, 066006 (2014).

27. E. S. Albert et al., "TRPV4 channels mediate the infrared laser-evoked response in sensory neurons," J Neurophysiol 107, 3227-3234 (2012).

28. V. Lumbreras et al., "Pulsed infrared radiation excites cultured neonatal spiral and vestibular ganglion neurons by modulating mitochondrial calcium cycling," J. Neurophysiol. 112, 1246-1255 (2014).

29. J. M. Cayce et al., "Infrared neural stimulation of human spinal nerve roots in vivo," Neurophotonics 2, 015007 (2015).

30. M. Chernov and A. W. Roe, "Infrared neural stimulation: a new stimulation tool for central nervous system applications," Neurophotonics 1, 011011 (2014).

31. M. M. Chernov, G. Chen, and A. W. Roe, "Histological assessment of thermal damage in the brain following infrared neural stimulation," Brain Stimul. 7, 476-482 (2014).

32. J. M. Cayce et al., "Infrared neural stimulation of primary visual cortex in non-human primates," Neuroimage 84, 181-190 (2014).

33. S. Tozburun et al., "Temperature-controlled optical stimulation of the rat prostate cavernous nerves," J. Biomed. Opt. 18, 067001 (2013).

34. S. Tozburun et al., "Continuous-wave laser stimulation of the rat prostate cavernous nerves using a compact and inexpensive all single mode optical fiber system," J. Endourol. 25, 1727-1731 (2011).

35. I. U. Teudt et al., "Optical stimulation of the facial nerve: a new monitoring technique?" Laryngoscope 117, 1641-1647 (2007).

36. B. Entwisle et al., "In vitro neuronal depolarization and increased synaptic activity induced by infrared neural stimulation," Biomed. Opt. Express 7(9), 3211-3219 (2016).

37. L. E. Moreno et al., "Infrared neural stimulation: beam path in the guinea pig cochlea," Hear. Res. 282, 289-302 (2011).

38. M. W. Jenkins et al., "Optical pacing of the adult rabbit heart," Biomed. Opt. Express 4(9), 1626-1635 (2013).

39. B. Xie, C. Dai, and H. Li, "Attenuated infrared neuron stimulation response in cochlea of deaf animals may associate with the degeneration of spiral ganglion neurons," Biomed. Opt. Express 6(6), 1990-2005 (2015).

40. X. Tan et al., "Radiant energy required for infrared neural stimulation," Sci. Rep. 5, 13273 (2015). 
41. X. Tan et al., "Temporal properties of inferior colliculus neurons to photonic stimulation in the cochlea," Physiol Rep 3, e12491 (2015).

42. J. E. Swett et al., "Sensory neurons of the rat sciatic nerve," Exp. Neurol. 114, 82-103 (1991).

43. J. M. Cayce et al., "Pulsed infrared light alters neural activity in rat somatosensory cortex in vivo," Neuroimage 57, 155-166 (2011).

44. J. M. Cayce et al., "Infrared neural stimulation of thalamocortical brain slices," IEEE J. Sel. Top. Quantum Electron. 16, 565-572 (2010).

45. H. K. Young et al., "Target structures for cochlear infrared neural stimulation," Neurophotonics 2, 025002 (2015).

46. R. G. McCaughey, C. Chlebicki, and B. J. F. Wong, "Novel wavelengths for laser nerve stimulation," Lasers Surg. Med. 42, 69-75 (2010).

47. M. Dautrebande et al., "In vivo photonic stimulation of sciatic nerve with a $1470 \mathrm{~nm}$ laser," Eur. J. Transl. Myol. 26, 6028 (2016).

48. Y. T. Wang et al., "Optical mapping of optically paced embryonic hearts," in 35th Annu. Int. Conf. IEEE Eng. Med. and Biol. Soc. (EMBC), IEEE, pp. 1623-1626 (2013).

49. E. J. Peterson and D. J. Tyler, "Motor neuron activation in peripheral nerves using infrared neural stimulation," J. Neural Eng. 11, 016001 (2014).

50. J. M. Cayce et al., "Calcium imaging of infrared-stimulated activity in rodent brain," Cell Calcium 55, 183-190 (2014).

51. A. I. Matic et al., "Behavioral and electrophysiological responses evoked by chronic infrared neural stimulation of the cochlea," PLoS One 8, e58189 (2013).

52. N. M. Fried et al., "Noncontact stimulation of the cavernous nerves in the rat prostate using a tunable-wavelength thulium fiber laser," J. Endourol. 22, 409-414 (2008).

53. N. M. Fried et al., "Laser stimulation of the cavernous nerves in the rat prostate, in vivo: optimization of wavelength, pulse energy, and pulse repetition rate," in 30th Annu. Int. Conf. IEEE Eng. Med. and Biol. Soc., IEEE, pp. 2777-2780 (2008).

54. S. Tozburun et al., "Infrared laser nerve stimulation as a potential diagnostic method for intra-operative identification and preservation of the prostate cavernous nerves," IEEE $J$. Sel. Top. Quantum Electron. 20, 299-306 (2014).

55. N. M. Fried et al., "Identification and imaging of the nerves responsible for erectile function in rat prostate, in vivo, using optical nerve stimulation and optical coherence tomography," IEEE J. Sel. Top. Quantum Electron. 13, 1641-1645 (2007).

56. V. Goyal et al., "Acute damage threshold for infrared neural stimulation of the cochlea: functional and histological evaluation," Anat. Rec. Adv. Integr. Anat. Evol. Biol. 295, 1987-1999 (2012).

57. J. D. Wells et al., "Optically mediated nerve stimulation: identification of injury thresholds," Lasers Surg. Med. 39, 513-526 (2007).

58. A. R. Duke et al., "Hybrid electro-optical stimulation of the rat sciatic nerve induces force generation in the plantarflexor muscles," J. Neural Eng. 9, 066006 (2012).

59. G. M. Hale and M. R. Querry, "Optical constants of water in the 200-nm to 200- $\mu$ m wavelength region," Appl. Opt. 12, 555 (1973).

60. J. M. Khosrofian and B. A. Garetz, "Measurement of a Gaussian laser beam diameter through the direct inversion of knife-edge data," Appl. Opt. 22, 3406 (1983).

61. E. D. Jansen et al., "Effect of pulse duration on bubble formation and laser-induced pressure waves during holmium laser ablation," Lasers Surg. Med. 18, 278-293 (1996).

62. A. D. Izzo et al., "Laser stimulation of the auditory nerve," Lasers Surg. Med. 38, 745-753 (2006).

63. A. R. Duke et al., "Spatial and temporal variability in response to hybrid electro-optical stimulation," J. Neural Eng. 9, 036003 (2012).

64. M. W. Jenkins et al., "Optical pacing of the embryonic heart," Nat. Photonics 4, 623-626 (2010).

65. A. E. Kirby and J. C. Middlebrooks, "Auditory temporal acuity probed with cochlear implant stimulation and cortical recording," J. Neurophysiol. 103, 531-542 (2010).

66. P. Julkunen et al., "Threshold curves for transcranial magnetic stimulation to improve reliability of motor pathway status assessment," Clin. Neurophysiol. 122, 975-983 (2011). 
67. M. Hermann, C. Hellebart, and M. Freissmuth, "Neuromonitoring in thyroid surgery: prospective evaluation of intraoperative electrophysiological responses for the prediction of recurrent laryngeal nerve injury," Ann. Surg. 240, 9-17 (2004).

68. J. M. Gibson and W. I. Welker, "Quantitative studies of stimulus coding in first-order vibrissa afferents of rats. 2. Adaptation and coding of stimulus parameters," Somatosens. Res. 1, 95-117 (1983).

69. M. S. Islam et al., "Extracting structural features of rat sciatic nerve using polarizationsensitive spectral domain optical coherence tomography," J. Biomed. Opt. 17, 056012 (2012).

70. A. D. Izzo et al., "Optical parameter variability in laser nerve stimulation: a study of pulse duration, repetition rate, and wavelength," IEEE Trans. Biomed. Eng. 54, 1108-1114 (2007).

71. A. D. Izzo et al., "Laser stimulation of auditory neurons: effect of shorter pulse duration and penetration depth," Biophys. J. 94, 3159-3166 (2008).

72. A. Vogel and V. Venugopalan, "Mechanisms of pulsed laser ablation of biological tissues," Chem. Rev. 103, 577-644 (2003).

73. N. Farah et al., "Holographically patterned activation using photo-absorber induced neuralthermal stimulation," J. Neural Eng. 10, 56004 (2013).

74. Y. Weissler, N. Farah, and S. Shoham, "Simulation of morphologically structured photothermal neural stimulation," J. Neural Eng. 14, 055001 (2017).

Graham Throckmorton received his BS degree in biomedical engineering and mathematics from Baylor University in 2016. He is currently a PhD candidate at Vanderbilt University in the Department of Biomedical Engineering, focusing on optical neuromodulation and label-free intraoperative nerve mapping.

Johnathan Cayce received his BS degree in biomedical engineering from the University of Alabama at Birmingham in 2006. He received his MS and PhD degrees in 2013 from Vanderbilt University, both in biomedical engineering. He is currently a clinical research scientist at DeRoyal Industries.

Zane Ricks received his BS degree in mechanical engineering from the University of Nevada, Reno, in 2010. He received his MS degree in biomedical engineering from Vanderbilt University in 2014. He is currently a GRE tutor at Varsity Tutors.

Wilson R. Adams received his BS degree in bioengineering from the University of Maine at Orono. He is currently a $\mathrm{PhD}$ candidate in biomedical engineering at the Vanderbilt Biophotonics Center focusing on fluorescence and nonlinear optical microscopy, neuromodulation, glial biology, and optical image processing in biomedicine.

Eric Duco Jansen received his MS (drs.) degree in medical sciences from the University of Utrecht, and his $\mathrm{MS}$ and $\mathrm{PhD}$ degrees in biomedical engineering from the University of Texas. He is currently a professor of biomedical engineering and neurosurgery at Vanderbilt University and is the associate dean for graduate studies in the School of Engineering. His research focus is on application of light, lasers, and optical technology in medicine and biology.

Anita Mahadevan-Jansen received her BS and MS degrees in physics from the University of Bombay and her MS and PhD degrees in biomedical engineering from the University of Texas. She is the Orrin H. Ingram Professor of biomedical engineering and a professor of neurological surgery at Vanderbilt University. Her research focus is on application of optical techniques for diagnosis of pathology, stimulation of neural tissue, and translation of optical technology for clinical applications. 